\title{
Mechanoresponsive Behavior of a Polymer-Embedded Red-Light Emitting Rotaxane Mechanophore
}

\author{
Tatsuya Muramatsu, ${ }^{\dagger}$ Yoshimitsu Sagara, ${ }^{,+, \neq}$Hanna Traeger, ${ }^{\S}$ Nobuyuki Tamaoki, ${ }^{\dagger}$ Christoph \\ Weder $^{*, \S}$ \\ ${ }^{\dagger}$ Research Institute for Electronic Science, Hokkaido University, N20, W1o, Kita-Ku, Sapporo, Hokkaido oo1-oo2o, \\ Japan \\ ${ }^{\ddagger}$ JST-PRESTO, Honcho 4-1-8, Kawaguchi, Saitama 332-0012, Japan \\ ${ }^{\S}$ Adolphe Merkle Institute, University of Fribourg, Chemin des Verdiers 4, CH-17oo Fribourg, Switzerland \\ Supporting Information Placeholder
}

\begin{abstract}
A red-light-emitting photoluminescent supramolecular mechanophore based on an interlocked molecular motif is presented. The rotaxane-based mechanophore contains a cyclic compound featuring a $\pi$-extended 4,4-difluoro-4bora-3a,4a-diaza-s-indacene (BODIPY) dye as a red emitter that was threaded onto a dumbbell-shaped molecule containing an electron-poor 1,4,5,8-naphthalenetetracarboxylic diimide quencher at its center. Through two aliphatic hydroxyl groups attached to the dumbbell and the cycle, the mechanophore was covalently embedded into the backbone of a thermoplastic polyurethane elastomer. The mechanophore is only weakly photoluminescent in solution, indicating that the BODIPY's emission is efficiently quenched. Solution-cast films of the rotaxane-containing polymer, by contrast, show an appreciable photoluminescence, which suggests that during film formation some of the emitting cycles are trapped in positions away from the quencher. Interestingly, the emission intensity could be significantly reduced by swelling the films with an organic solvent and the emission increased again upon drying, suggesting that such solvent plasticization causes a reversible rearrangement. In both, dry and solvent-swollen films, uniaxial deformation caused a significant, reversible increase of the emission intensity, on account of mechanically induced shuttling of the emitters away from and back to the quenchers. It is shown that the properties of the polymer can be tuned by the solvent, and that such plasticizing extends the small palette of approaches that allow modification of the activation stress of a given materials system.
\end{abstract}

KEYWORDS: mechanophore, rotaxane, BODIPY, mechanoresponsive luminescence, supramolecular mechanophore

\section{- INTRODUCTION}

The visualization and quantification of mechanical stresses in polymers represents a technologically important but challenging task. ${ }^{1-3}$ As was demonstrated first a decade ago, mechanochromic mechanophores motifs that display mechanically activated photophysical changes and can be integrated into polymers - represent an attractive approach to solve this problem. ${ }^{4}$ In the meantime, many color-changing mechanophores and corresponding motifs which change their photoluminescence properties have been investigated. ${ }^{4-21}$ While the latter require auxiliary means for detection, a change of the emission color and/or intensity can often be detected more easily than an absorption color change. ${ }^{22}$ Most of the fluorescent mechanophores reported so far, emit in the blue and green wavelength region. For instance, nonemissive anthracene-maleimide Diels-Alder adducts exhibit blue emission after activation ${ }^{5-8}$ and a bathochromic shift of the emission band can be achieved by extension of $\pi$-conjugation of the anthracene moiety. ${ }^{9} 1,2-$ Dioxetane, which is a chemiluminescent mechanophore, exhibits blue emission upon activation. ${ }^{10-12} \mathrm{~A}$ mechanophore based on an aliphatic ester of 2-(2'hydroxyphenyl)benzoxazole was shown to switch from blue to green emission after cleavage of the ester bond, which enabled an excited-state intramolecular proton transfer mechanism. ${ }^{13}$ Finally, the green fluorescent dithiomaleimide motif was used as a mechanically responsive turn-off motif. ${ }^{14}$

While the number of papers dealing with blue- or greenemissive mechanophores is steadily increasing, reports on orange or red-light emitting systems are still limited. However, such systems are useful, in particular in applications that require the simultaneous use of several mechanophores, such as for example in bioimaging, where several fluorescent dyes are utilized to visualize living cells and their functions, ${ }^{22}$ or to create white light. ${ }^{21}$ So far, rhodamine derivatives, ${ }^{15}$ an Eu ${ }^{3+}$ complex,$^{16}$ several copper complexes $^{17,18}$ and a rotaxane having $\pi$-extended 4(dicyanomethylene)-2-methyl-6-(4-dimethylaminostyryl)$4 H$-pyran (DCM) group as the emitter ${ }^{21}$ were used as orange or red-light emitting mechanophores that can visualize mechanical stress when embedded in a polymer. In these motifs, the scission of a covalent bond, the dissociation between $\mathrm{Eu}^{3+}$ ions and a coordinating ligand, 
a change of the ligand exchange rate, and the shuttling function of rotaxanes were exploited to change redemissive character of the respective motif. Red emission was also observed from a merocyanine that was accessed by mechanically induced ring-opening of a spiropyran, ${ }^{4,20}$ although the quantum yield was low..$^{9,23}$

We recently reported supramolecular rotaxane-based mechanoluminophores composed of a cycle with an appended luminophore and a dumbbell-shaped molecule containing an electronically matched quencher and two stopper groups. ${ }^{19,21}$ Linear polyurethane elastomers containing these motifs exhibit instantly reversible ON/OFF switching of their photoluminescence and the emission color could be tuned from blue to green to orange via the choice of the emitter. The activation does not involve any bond cleavage, but relies instead on the temporary separation of the luminophores and the quencher. As a result, the activation energy is low, the process is completely and instantly reversible, and the activation is fast and specific to mechanical force. Here we demonstrate that this approach can be utilized to create a red-light emitting mechanophore. Interestingly, an elastic polyurethane (PU) containing the mechanophore shows non-negligible red emission in the absence of any stress, which contrasts the behavior of previously investigated PUs with other rotaxane-containing mechanoluminophores. ${ }^{19,21}$ We also show that the properties of the redemissive polyurethane can be tuned by incorporation of a plasticizing solvent, and thereby extend the small palette of approaches that allow modification of the threshold stress required for the activation of a given mechanophore in a given polymer system. ${ }^{24}$

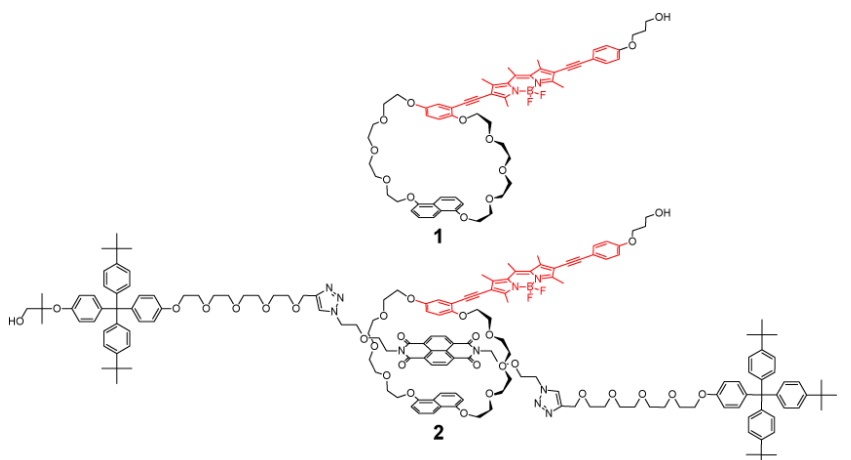

Figure 1. Molecular structures of the BODIPY-based cyclic compound $\mathbf{1}$ and rotaxane-based supramolecular mechanophore 2.

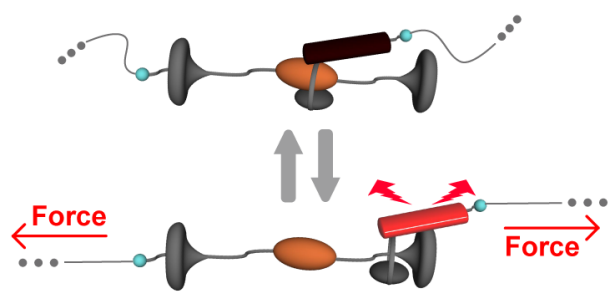

Figure 2. Schematic illustration of the working mechanism of the red-light emitting, rotaxane-based mechanophore 2.

- RESULTS AND DISCUSSION
A $\pi$-extended 4,4-difluoro-4-bora-3a,4a-diaza-s-indacene (BODIPY) dye was used as the red emitter in this study. BODIPYs represent a well-known family of fluorophores that exhibit usually a high quantum efficiency and allow facile molecular structure variations, which has led researchers to investigate a large number of derivatives that cover a broad range of emission colors. ${ }^{25}$ We first synthesized the red-light-emitting $\pi$-extended BODIPY derivative $\mathbf{1}$, in which the dye forms part of a cyclic structure composed of a naphthalene group and two tetraethylene glycol chains (Figure 1 ). ${ }^{26}$ Phenylethynyl groups were introduced at the 2- and 6-positions of the BODIPY to extend its $\pi$-conjugation. ${ }^{27-29}$ The rotaxanebased supramolecular mechanophore $\mathbf{2}$ was then synthesized through a 1,3-dipolar cycloaddition-type click reaction. ${ }^{30}$ The product was characterized to satisfaction by ${ }^{1} \mathrm{H}$ and ${ }^{13} \mathrm{C}$ NMR spectroscopy and high-resolution ESI-MS (See Supporting Information). The dumbbell-shaped molecule, which constitutes the second part of the rotaxane, contains an electron-poor 1,4,5,8-naphthalenetetracarboxylic diimide $(\mathrm{NpI})^{31-34}$ as a matching quencher and two tetraphenyl methane units featuring three tertbutyl groups as stoppers. ${ }^{35-37}$ The emitter and one of stopper groups were equipped with hydroxyl-groups in order to covalently incorporate the mechanophore into polymers. ${ }^{19,21}$ As illustrated in Figure 2, weak emitterquencher interactions should place the emitter preferentially near the quencher in the absence of mechanical force, so that the red luminescence is quenched by the NpI group. The application of mechanical stress transduced to the mechanophore through polymer chains was expected to cause sliding of the cyclic emitter along the axle and thereby a turn-on of the red luminescence. ${ }^{19,21}$

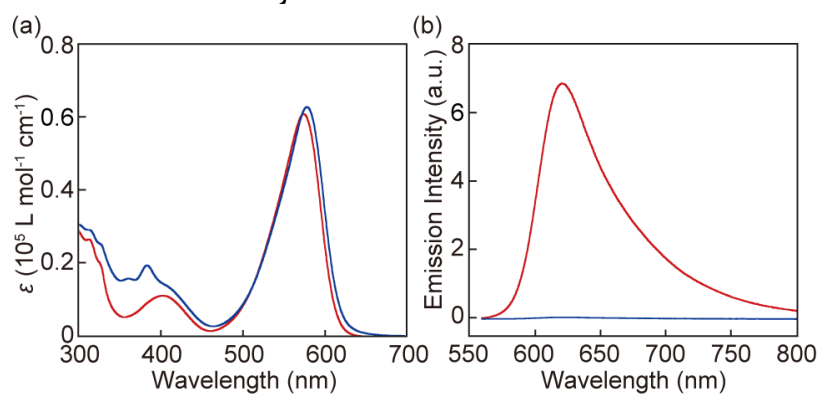

Figure 3. (a) Optical absorption and (b) photoluminescence spectra of cyclic compound $\mathbf{1}$ (red) and rotaxane $\mathbf{2}$ (blue) in chloroform (recorded at $\mathrm{c}=1.0 \times 10^{-5} \mathrm{M}$ ). The photoluminescence spectra were recorded with excitation light of $550 \mathrm{~nm}$.

Absorption and photoluminescence measurements were performed before the mechanophore was introduced into a polymer. A solution of cyclic compound $\mathbf{1}$ in chloroform $\left(1.0 \times 10^{-5} \mathrm{M}\right)$ displays an absorption band with a maximum at $575 \mathrm{~nm}$ (Figure 3a, red line). A slight red shift of the absorption band was observed after rotaxane formation. The increased absorbance between 350 and $400 \mathrm{~nm}$ after rotaxane formation is ascribed to the NpI group in the rod molecule. The chloroform solution of $\mathbf{1}$ exhibits bright red photoluminescence and the emission peak features a maximum at $620 \mathrm{~nm}$ (Figure $3 \mathrm{~b}$ ). The quantum yield of $\mathbf{1}$ is 
0.32 and this value is similar to those of reported redemissive BODIPY derivatives. ${ }^{25,28,29}$ As expected, little photoluminescence was observed from a solution of rotaxane 2, which confirms that the photoluminescence of the red emitter was efficiently quenched by the NpI group. Indeed, the quantum yield decreased from 0.32 to below 0.01 .

The mechanophore $\mathbf{2}$ was covalently introduced into a thermoplastic polyurethane elastomer by a polyaddition reaction with poly(tetrahydrofuran), 4,4-methylenebis(phenylisocyanate), and 1,4-butanediol. This matrix was chosen, as its mechanical behavior proved to be useful to investigate the reversible mechanoactivation of other mechanophores. ${ }^{19,21}$ The concentration of the mechanophore in the monomer feed was ca. $0.2 \mathrm{wt} \%$. On account of its low concentration, the incorporation of the mechanophore in the polymer could not be identified by ${ }^{1} \mathrm{H}$ NMR spectroscopy (Figure S2). However, optical absorption and emission spectra of polyurethane 2-PU reveal the presence of the mechanophore and reveal that embedding 2 into the macromolecules has almost no effect on the photophysical properties of the rotaxane in solution (Figure $\mathrm{S}_{3}$ ). Thermogravimetric analysis (TGA) (Figure 44 ) and differential scanning calorimetric (DSC) measurements (Figure $\mathrm{S}_{5}$ ) revealed that 2-PU has similar thermal properties as related polyurethanes that we previously reported, ${ }^{19,21,38,39}$ i.e., the DSC shows a broad peak corresponding to melting or disordering of the hard segments around $180{ }^{\circ} \mathrm{C}$, while the TGA scan shows that decomposition gradually occurs above $300^{\circ} \mathrm{C}$. After $80-100$ $\mu \mathrm{m}$ thin films of 2-PU were prepared by solution casting, the specimen were subjected to dynamic mechanical analysis (DMA) (Figure S6). The DMA traces show a glassy regime below $-60{ }^{\circ} \mathrm{C}$ and a rubbery plateau that reaches from above $T_{g}$ to the failure temperature of ca. $180{ }^{\circ} \mathrm{C}$. Uniaxial tensile tests (Figure $\mathrm{S}_{7}$, Table $\mathrm{S}_{1}$ ) reveal a Young's modulus of $13 \pm 3 \mathrm{MPa}$ and show that the introduction of the (small amount of) rotaxane has not changed the mechanical properties of the polymer. ${ }^{19,21}$

Contrary to our initial expectations, as-prepared, solution-cast 2-PU films exhibit weak red photoluminescence under ambient conditions $(\Phi=0.04)$, although the THF solution of this polymer is hardly emissive (Figure $\mathrm{S} 3 \mathrm{~b}$ ). This behavior mirrors the one of a solution-cast polymer containing a rotaxane-based mechanophore with 4,7-bis(phenylethynyl)-2,1,3-benzothiadiazole as the green emitter, which also shows faint photoluminescence. ${ }^{19}$ To explore the origin of this behavior, we prepared physical blends of mechanophore $\mathbf{2}$ and a similar reference polyurethane made without a covalently incorporated mechanophore (2inPU). Solution-cast zinPU films also show red emission (Figure S8), which confirms that this effect is not related to any mechanical force that is transduced via the polymer. The behavior of both, the zinPU reference blend as well as the mechano-active polymer 2-PU, is consistent with kinetic trapping of arrangements in which the emitters are placed away from the quenchers upon drying. Such trapping is possible because of the weak interaction between the luminophore and the quencher, and the limited mobility in the dried state. The fact that the shape of the emission spectra of the luminophore solution (Figure 3a), the reference PU films (inPU and zinPU, Figures S8,S9), and 2-PU films are very similar suggests that the PU matrix does not influence the photophysical properties of the BODIPY-based fluorophore. When 2-PU films were uniaxially stretched, the emission intensity initially decreased but then gradually increased when the strain $\lambda=\left(\mathrm{L}-\mathrm{L}_{0}\right) / \mathrm{L}_{0}$ exceeded 200\% (Figure 4a, Movie $\mathrm{S}_{1}$ ). The fact that reference films based on a physical blend of the reference polyurethane and compounds 1 (1inPU) or 2 (2inPU) show only a decrease of the emission intensity when stretched (Figure S8,S9) suggests that the intensity decrease observed for 2-PU at low strains can be attributed to a decrease of the film thickness, before additional mechanophores are activated at higher strains and the emission intensity increases. The reference experiments further highlight that the covalent incorporation of the mechanophore into the polymer chains is a requirement for its mechanical activation.

The mechanoresponsive behavior of 2-PU was also investigated by sonomechanochemical experiments, which are routinely used to probe the mechanically induced scission of covalent bonds (Figure Sio). Thus, when THF solutions of 2-PU were sonicated over the course of $60 \mathrm{~min}$, a gradual decrease of the molecular weight and a concomitant increase of the photoluminescence intensity were observed. These results indicate the scission of covalent bonds in the mechanophore or de-threading of the cycle upon sonication.
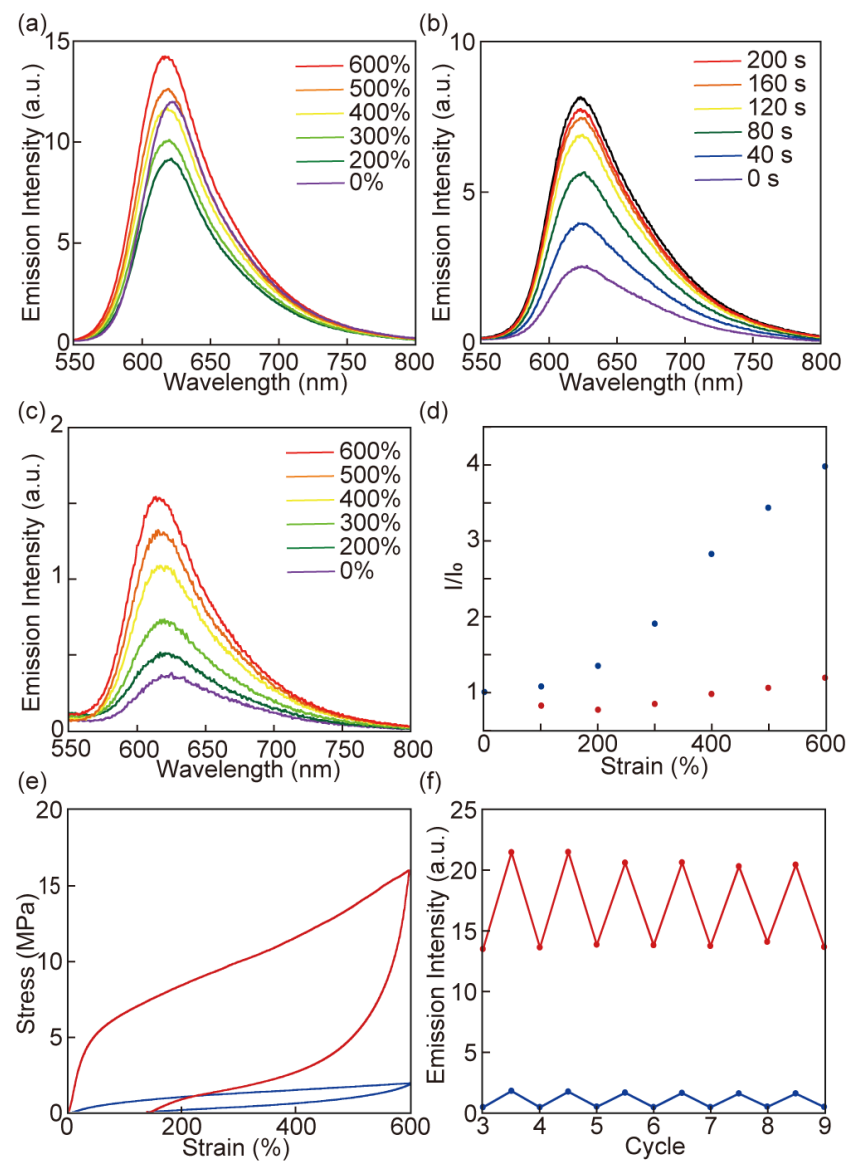
Figure 4. (a) Emission spectra of a solution-cast 2-PU film recorded upon uniaxial tensile deformation to the strain indicated. (b) Emission spectra of a 2-PU film swollen with rapidly evaporating ethyl acetate. The data collection was started just after removing the film from the solvent $(t=0 \mathrm{~s})$ and spectra were acquired after maintaining the sample for the times indicated under ambient conditions. The black line represents the emission spectrum of a solution-cast 2-PU film recorded under the same conditions. (c) Emission spectra of a phenylacetonitrile swollen 2-PU film recorded upon uniaxial tensile deformation to the strain indicated. (d) Plots of the relative emission intensity $\mathrm{I} / \mathrm{I}_{\mathrm{o}}$ of a solution-cast (red) and a phenylacetonitrile swollen (blue) 2-PU film as a function of strain. (e) Stress-strain curves of a solution-cast (red) and a phenylacetonitrile swollen (blue) 2-PU film. (f) Emission intensities recorded upon deformation for a solution-cast (red) and a phenylacetonitrile swollen (blue) 2-PU film over 9 cycles. The emission spectra were recorded with excitation at $385 \mathrm{~nm}$ and emission intensities in (d) and ( $f$ ) were recorded at 620 nm.

Interestingly, swelling the 2-PU films with organic solvents leads to a decrease of the initial emission intensity (Table S2). This effect was observed when the films were immersed in ethyl acetate, chloroform, acetone, and phenylacetonitrile, whereas $\mathrm{MeOH}, \mathrm{EtOH}$, hexane, and water did not swell the polymer sufficiently to change the emission intensity significantly. The swelling-induced photoluminescence reduction was fully reversible, i.e., when the solvent was evaporated under ambient conditions, the emission intensity gradually increased (Figure $4 \mathrm{~b}$ ), suggesting that the effect is related to the increased mobility upon plasticization: in the presence of the solvent the shuttling of the cycle along the axle is restored, the average distance between quencher and emitter is decreased, and the fluorescence is reduced. In order to conduct quantitative experiments with solventswollen films under ambient conditions, we swelled 2-PU with phenylacetonitrile, as the high boiling point $\left(233^{\circ} \mathrm{C}\right)$ renders the evaporation of this solvent slow. Upon swelling with phenylacetonitrile, the quantum yield of 2-PU decreased from 0.04 to below 0.01. As shown in Figure 4c, straining the phenylacetonitrile swollen 2-PU film to a strain of $600 \%$ leads to an increase of the emission intensity of ca. $400 \%\left(\lambda_{\mathrm{em}}=617 \mathrm{~nm}\right)$. Plots of the relative emission intensities, i.e., the ratio of the emission intensities of the stretched (I) and unstretched sample ( $\left.\mathrm{I}_{\mathrm{o}}\right)$ recorded at $620 \mathrm{~nm}$, of the solvent-cast 2-PU film and the phenylacetonitrile swollen film as a function of strain, show that the solvent-containing film exhibits a much larger contrast (Figure $4 \mathrm{~d}$ ), which coincides with the observed mechanoresponsive luminescence displayed in Movie S2. Stress-strain curves reveal that the 2-PU films becomes much softer and weaker upon swelling and the stress required to achieve deformation and trigger the mechanoresponse is significantly reduced (Figure 4e). In the case of the dry material, a stress of $16 \mathrm{MPa}$ is required to achieve a strain of $600 \%$, while this value is reduced to 1.9 MPa upon swelling. Such plasticization (which could likely be achieved with non-volatile plasticizers) allows one to modify the mechanoresponse of a given polymer and one can tailor the properties easily in this way.

The hystereses observed in the strain-stress curves upon uniaxial elongation and stress release (Figure 4e) are also reflected in the corresponding emission intensities (Figure S11). Samples which had been swelled with phenylacetonitrile and were subsequently dried in vacuo for 1 day show a similarly small change of the relative emission intensity upon stretching (Figure S12) as the dry films after casting (Figure 4a), although the original mechanical properties were not completely restored (Figure S13), perhaps due to residual solvent. The emission intensities recorded during several loading-unloading cycles with a strain of $600 \%$ (Figure $4 \mathrm{f}$ ) reveal reversible and rapid switching between constant states of low and high emission intensity for both, solution-cast and phenylacetonitrile swollen 2-PU films. The absolute emission intensities of the solvent-containing films are lower than those of the dry sample, because the applied mechanical stress and the quantum yield of the mechanophore are both lower. As mentioned before, the quantum yield of $\mathbf{1}$ in chloroform is 0.32 , while the value decreases to 0.19 in acetonitrile.

To further probe the proposed working mechanism at play in 2-PU films, as depicted in Figure 2, emission lifetime measurements were performed (Figure 5). The reference 1 inPU gave a decay profile that can be fitted with a bi-exponential decay function with calculated emission lifetimes of 2.6 and $3.6 \mathrm{~ns}$. This suggests that the naphthalene group of compound $\mathbf{1}$ affects the BODIPY luminophores, resulting in two emission species. Similar multi-lifetime emission decays were also observed for other luminescent cyclophanes featuring 1,5-disubstituted naphthalene moieties. ${ }^{40,41}$ Emissive states with shorter lifetimes were observed for solution-cast 2-PU films. This change in emission decay is related to quenching by the NpI group, and reflects that in the solution-cast 2-PU films, the latter resides, on average, in the vicinity of the cycle. Upon deformation to a strain of $600 \%$, the emission lifetime becomes longer, indicative of an increase of the population of red luminophores that are located away from the NpI groups. Together with the emission intensities, these results support the working mechanism depicted in Figure 2. The solvent-swollen 2-PU film also shows a strain-induced increase of the emission lifetime, although both lifetimes are shorter than solvent-free samples, on account of solvent effects.

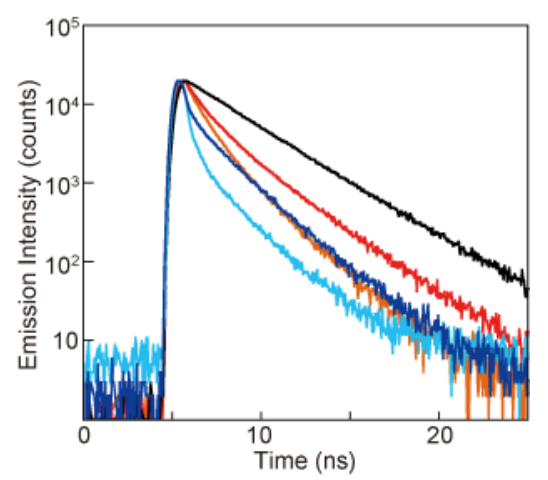

Figure 5. Emission decay profiles of a solution-cast 2-PU film recorded before and after uniaxial deformation to a strain of 
$600 \%$ (orange and red) and of a phenylacetonitrile swollen 2PU film recorded before and after uniaxial deformation to a strain of $600 \%$ (light and dark blue). The emission decay profile of $\mathbf{1}$ in chloroform (black line) is also shown. All decay profiles were monitored at $620 \mathrm{~nm}$, with excitation at $590 \mathrm{~nm}$.

Thus, molecular shuttling effects play a pivotal role in the swelling-induced changes of the mechanoresponsive luminescence exhibited by 2-PU. Upon solvent casting, some of the red-light emitters are trapped in thermodynamically unfavorable locations towards the ends of the dumbbell's axle, as a result of the weak quencher-cycle interactions. After solvent swelling, the molecular mobility is increased and a portion of the kinetically trapped emitters can slide towards the $\mathrm{NpI}$ moiety where it is localized on account of (weak) interactions. Consequently, the emission intensity is reduced upon swelling and the emission contrast between unstretched and stretched samples becomes much larger than in the solvent-free material.

\section{- CONCLUSIONS}

In summary, a new type of red-light emitting mechanophore was accessed through a supramolecular approach. The BODIPY-containing rotaxane is only weakly photoluminescent in solution, indicating that the emitter is efficiently quenched by the diimide present in the rotaxane's axle. By contrast, solution-cast films of the rotaxane-containing polyurethane prepared, show an appreciable red luminescence, which suggests that during film formation a portion of the emitting cycles are trapped away from the quencher. However, the fluorescence intensity of the solid material, as well as its mechanical strength and stiffness, can be significantly reduced by swelling the polymer films with an organic solvent. In all cases, uniaxial deformation increased the emission intensity, on account of the mechanically induced shuttling of the rotaxane. Compared to the red-emissive, mechano-responsive Eu complexes investigated previously, ${ }^{16}$ the present mechanophore shows instantly reversible changes when covalently introduced into polyurethane chains and the activation of rotaxane-based mechanophore requires weaker forces, as no scission of coordination bond is needed. Because red-light emitting mechanophores are very useful when it comes to biological application to visualize small mechanical forces, other approaches to develop red-emissive supramolecular mechanophores as well as efforts to integrate and investigate rotaxane mechanophores in other polymer matrices are undergoing in our groups.

\section{ASSOCIATED CONTENT}

\section{Supporting Information}

The Supporting Information is available free of charge on the ACS Publications website at DOI: 10.1021/acsami.XXXXXXX.

Experimental procedure and additional data (PDF).
Movie S1: Representative movie of the mechanoresponsive luminescent behavior upon cyclic stretching of the 2-PU film.

Movie S2: Representative movie of the swelling effect and subsequent mechanoresponsive luminescent behavior upon cyclic stretching the 2PU film.

\section{- AUTHOR INFORMATION}

\section{Corresponding Author}

*Y.S. sagara@es.hokudai.ac.jp

${ }^{*}$ C.W. christoph.weder@unifr.ch

ORCID

Yoshimitsu Sagara: oooo-0003-2502-3041

Nobuyuki Tamaoki: oooo-0oo3-1079-7087

Christoph Weder: oooo-ooo1-7183-1790

Notes

The authors declare no competing financial interest.

\section{- ACKNOWLEDGMENT}

We thank Ms. M. Yamada for HRMS measurements and Prof. Y. Urano for emission quantum yield measurements. This work was performed under the International Cooperative Research Program of "Dynamic Alliance for Open Innovation Bridging Human, Environment and Materials" in "Network Joint Research Center for Materials and Devices". This work was supported by the Japan Science Technology Agency (JST), PRESTO (No. JPMJPRi7P6) and the Tanaka Rubber Science and Technology Award. This work was also supported by JSPS Core-to-Core Program, A. Advanced Research Networks. This work also benefitted from support from the Adolphe Merkle Foundation and the Swiss National Science Foundation through the Swiss National Center of Competence in Research Bio-Inspired Materials.

\section{- REFERENCES}

(1) Caruso, M. M.; Davis, D. A.; Shen, Q.; Odom, S. A.; Sottos, N. R.; White, S. R.; Moore, J. S. Mechanically-Induced Chemical Changes in Polymeric Materials. Chem. Rev. 2009, 109, $5755^{-5798}$

(2) Li, J.; Nagamani, C.; Moore, J. S. Polymer Mechanochemistry: From Destructive to Productive. Acc. Chem. Res. 2015, 48, 2181-2190.

(3) Calvino, C.; Neumann, L.; Weder, C.; Schrettl, S. Approaches to Polymeric Mechanochromic Materials. J. Polym. Sci., Part A: Polym. Chem. 2017, 55, 640-652.

(4) Davis, D. A.; Hamilton, A.; Yang, J.; Cremar, L. D.; Van Gough, D.; Potisek, S. L.; Ong, M. T.; Braun, P. V.; Martinez, T. J.; White, S. R.; Moore, J. S.; Sottos, N. R. Force-Induced Activation of Covalent Bonds in Mechanoresponsive Polymeric Materials. Nature 2009, 459, 68-72.

(5) Sulkanen, A. R.; Sung, J.; Robb, M. J.; Moore, J. S.; Sottos, N. R.; Liu, G. Spatially Selective and Density-Controlled Activation of Interfacial Mechanophores. J. Am. Chem. Soc. 2019, $141,4080-4085$.

(6) Sung, J.; Robb, M. J.; White, S. R.; Moore, J. S.; Sottos, N. R. Interfacial Mechanophore Activation Using Laser-Induced Stress Waves. J. Am. Chem. Soc. 2018, 140, 5000-5003.

(7) Li, H.; Göstl, R.; Delgove, M.; Sweeck, J.; Zhang, Q.; Sijbesma, R. P.; Heuts, J. P. A. Promoting Mechanochemistry of 
Covalent Bonds by Noncovalent Micellar Aggregation. ACS Macro Lett. 2016, 5, 995-998.

(8) Gossweiler, G. R.; Hewage, G. B.; Soriano, G.; Wang, Q.; Welshofer, G. W.; Zhao, X.; Craig, S. L. Mechanochemical Activation of Covalent Bonds in Polymers with Full and Repeatable Macroscopic Shape Recovery. ACS Macro Lett. 2014, 3 , 216-219.

(9) Göstl, R.; Sijbesma, R. P. $\pi$-Extended Anthracenes as Sensitive Probes for Mechanical Stress. Chem. Sci. 2016, 7, 370375 .

(10) Clough, J. M.; van der Gucht, J.; Sijbesma, R. P. Mechanoluminescent Imaging of Osmotic Stress-Induced Damage in a Glassy Polymer Network. Macromolecules 2017, 50, 2043-2053.

(11) Ducrot, E.; Chen, Y.; Bulters, M.; Sijbesma, R. P.; Creton, C. Toughening Elastomers with Sacrificial Bonds and Watching Them Break. Science 2014, 344, 186-189.

(12) Chen, Y.; Spiering, A. J. H.; Karthikeyan, S.; Peters, G. W. M.; Meijer, E. W.; Sijbesma, R. P. Mechanically Induced Chemiluminescence From Polymers Incorporating a 1,2Dioxetane Unit in the Main Chain. Nat. Chem. 2012, 4, 559-562.

(13) Karman, M.; Verde-Sesto, E.; Weder, C. Mechanochemical Activation of Polymer-Embedded Photoluminescent Benzoxazole Moieties. ACS Macro Lett. 2018, 7, 1028-1033.

(14) Karman, M.; Verde-Sesto, E.; Weder, C.; Simon, Y. C. Mechanochemical Fluorescence Switching in Polymers Containing Dithiomaleimide Moieties. ACS Macro Lett. 2018, 7, 1099-1104.

(15) Wang, T.; Zhang, N.; Dai, J.; Li, Z.; Bai, W.; Bai, R. Novel Reversible Mechanochromic Elastomer with High Sensitivity: Bond Scission and Bending-Induced Multicolor Switching. ACS Appl. Mater. Interfaces 2017, 9, 11874-11881.

(16) Balkenende, D. W. R.; Coulibaly, S.; Balog, S.; Simon, Y. C.; Fiore, G. L.; Weder, C. Mechanochemistry with Metallosupramolecular Polymers. J. Am. Chem. Soc. 2014, 136, 10493-10498.

(17) Filonenko, G. A.; Khusnutdinova, J. R. Dynamic Phosphorescent Probe for Facile and Reversible Stress Sensing. Adv. Mater. 2017, 29, 1700563.

(18) Filonenko, G. A.; Lugger, J. A. M.; Liu, C.; van Heeswijk, E. P. A.; Hendrix, M. M. R. M.; Weber, M.; Müller, C.; Hensen, E. J. M.; Sijbesma, R. P.; Pidko, E. A. Tracking Local Mechanical Impact in Heterogeneous Polymers with Direct Optical Imaging. Angew. Chem., Int. Ed. 2018, 57, 16385-16390.

(19) Sagara, Y.; Karman, M.; Verde-Sesto, E.; Matsuo, K.; Kim, Y.; Tamaoki, N.; Weder, C. Rotaxanes as Mechanochromic Fluorescent Force Transducers in Polymers. J. Am. Chem. Soc. 2018, 140, 1584-1587.

(20) Kim, T. A.; Robb, M. J.; Moore, J. S.; White, S. R.; Sottos, N. R. Mechanical Reactivity of Two Different Spiropyran Mechanophores in Polydimethylsiloxane. Macromolecules 2018, 51, 9177-9183.

(21) Sagara, Y.; Karman, M.; Seki, A.; Pannipara, M.; Tamaoki, N.; Weder, C.; Rotaxane-based Mechanophores Enable Polymers with Mechanically Switchable White Photoluminescence; ACS Cent. Sci. 2019, 5, 874-881.

(22) Ueno, T.; Nagano, T. Fluorescent Probes for Sensing and Imaging. Nat. Methods 2011, 8, 642-645.

(23) Chibisov, A. K.; Görner, H. Photoprocesses in Spiropyran-Derived Merocyanines. J. Phys. Chemi. A 1997, 101, 4305-4312.

(24) Kida, J.; Imato, K.; Goseki, R.; Aoki, D.; Morimoto, M.; Otsuka, H. The Photoregulation of a Mechanochemical Polymer Scission. Nature Commun. 2018, 9, 3504.
(25) Loudet, A.; Burgess, K. BODIPY Dyes and Their Derivatives: Syntheses and Spectroscopic Properties. Chem. Rev. 2007, 107, 4891-4932.

(26) Bruns, C. J.; Basu, S.; Stoddart, J. F. Improved Synthesis of 1,5-Dinaphtho[38]crown-10. Tetrahedron Lett. 2010, 51, 983986.

(27) Rödle, A.; Ritschel, B.; Mück-Lichtenfeld, C.; Stepanenko, V.; Fernández, G. Influence of Ester versus Amide Linkers on the Supramolecular Polymerization Mechanisms of Planar BODIPY Dyes. Chem. Eur. J. 2016, 22, 15772-15777.

(28) Liras, M.; Iglesias, M.; Sánchez, F. Conjugated Microporous Polymers Incorporating BODIPY Moieties as LightEmitting Materials and Recyclable Visible-Light Photocatalysts. Macromolecules 2016, 49, 1666-1673.

(29) Bonardi, L.; Ulrich, G.; Ziessel, R. Tailoring the Properties of Boron-Dipyrromethene Dyes with Acetylenic Functions at the 2,6,8 and 4-B Substitution Positions. Org. Lett. 2008, 10, 2183-2186.

(30) Rostovtsev, V. V.; Green, L. G.; Fokin, V. V.; Sharpless, K. B. A Stepwise Huisgen Cycloaddition Process: Copper(I)Catalyzed Regioselective "Ligation" of Azides and Terminal Alkynes. Angew. Chem., Int. Ed. 2002, 41, 2596-2599.

(31) Jacquot de Rouville, H.-P.; Iehl, J.; Bruns, C. J.; McGrier, P. L.; Frasconi, M.; Sarjeant, A. A.; Stoddart, J. F. A Neutral Naphthalene Diimide [2]Rotaxane. Org. Lett. 2012, 14, 5188-5191.

(32) Choudhary, U.; Northrop, B. H. Rotaxanes and Biofunctionalized Pseudorotaxanes via Thiol-Maleimide Click Chemistry. Org. Lett. 2012, 14, 2082-2085.

(33) Cougnon, F. B. L.; Jenkins, N. A.; Pantoş, G. D.; Sanders, J. K. M. Templated Dynamic Synthesis of a [3]Catenane. Angew. Chem., Int. Ed. 2012, 51, 1443-1447.

(34) Hamilton, D. G.; Davies, J. E.; Prodi, L.; Sanders, J. K. M. Synthesis, Structure and Photophysics of Neutral $\pi$-Associated [2]Catenanes. Chem. Eur. J. 1998, 4, 608-620.

(35) Gibson, H. W.; Lee, S. H.; Engen, P. T.; Lecavalier, P.; Sze, J.; Shen, Y. X.; Bheda, M. New Triarylmethyl Derivatives: "Blocking Groups" for Rotaxanes and Polyrotaxanes. J. Org. Chem. 1993, 58, 3748-3756.

(36) Dichtel, W. R.; Miljanić, O. Š.; Spruell, J. M.; Heath, J. R.; Stoddart, J. F. Efficient Templated Synthesis of Donor-Acceptor Rotaxanes Using Click Chemistry. J. Am. Chem. Soc. 2006, 128, 10388-10390.

(37) Gong, C.; Gibson, H. W. Synthesis and Characterization of a Polyester/Crown Ether Rotaxane Derived from a Difunctional Blocking Group. Macromolecules 1996, 29, 7029-7033.

(38) Ayer, M. A.; Simon, Y. C.; Weder, C. Azo-Containing Polymers with Degradation On-Demand Feature. Macromolecules 2016, 49, 2917-2927.

(39) Crenshaw, B. R.; Weder, C. Self-Assessing Photoluminescent Polyurethanes. Macromolecules 2006, 39, 9581-9589.

(40) Sagara, Y.; Seki, A.; Kim, Y.; Tamaoki, N. Linearly Polarized Photoluminescence From an Asymmetric Cyclophane Showing Thermo- and Mechanoresponsive Luminescence. J. Mater. Chem. C 2018, 6, 8453-8459.

(41) Sagara, Y.; Weder, C.; Tamaoki, N. Asymmetric Cyclophanes Permit Access to Supercooled Nematic Liquid Crystals with Stimulus-Responsive Luminescence. Chem. Mater. 2017, 29, 6145-6152. 
Mechanoresponsive Behavior of a Polymer-Embedded Red-Light Emitting Rotaxane Mechanophore

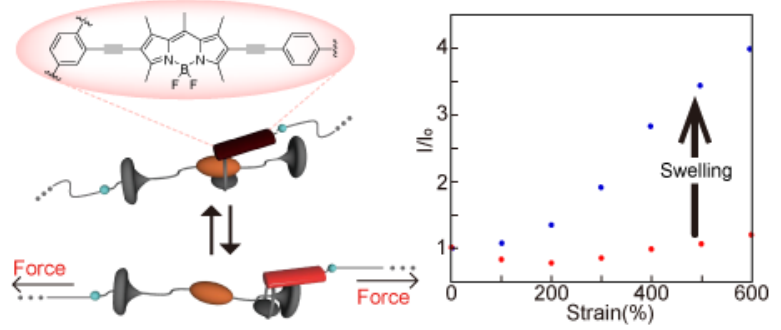

